\title{
On the Boundedness and Skew-Symmetric Properties of the Inertia and Coriolis Matrices for Vehicle-Manipulator Systems
}

\author{
Pål J. From* Ingrid Schjølberg** Jan T. Gravdahl* \\ Kristin Y. Pettersen* Thor I. Fossen* \\ * Department of Engineering Cybernetics, NTNU, Trondheim, Norway \\ ** Applied Cybernetics, SINTEF, Trondheim, Norway
}

\begin{abstract}
This paper addresses the boundedness property of the inertia matrix and the skewsymmetric property of the Coriolis matrix for vehicle-manipulator systems. These properties are widely used in Lyapunov-based stability proofs and are therefore important to identify. For example, the skew-symmetric property does not depend on the system at hand, but on the choice of parameterisation of the Coriolis matrix, which is not unique. It is the authors' experience that many researchers take this assumption for granted without taking into account that there exist several parameterisations for which this is not true. In fact, most researchers refer to references that do not show this property for vehicle-manipulator systems, but for other systems such as single rigid bodies or manipulators on a fixed base. As a result, the otherwise rigorous stability proofs fall apart. In this paper we point out several references that are widely used, but that do not show this property and we refer to the correct references. As most references on this topics are not easily accessible, we also give the correct proofs for commonly used parameterisations of the Coriolis matrix and thus provide a proof for future reference.

The same is the case for the boundedness property of the inertia matrix which for a bad choice of state variables will not necessarily hold. This can be solved by deriving the dynamics for vehiclemanipulator systems in terms of quasi-velocities, which allows us to describe the dynamics without the presence of the Euler angle singularities that normally arise in vehicle-manipulator dynamics. To the authors' best knowledge we derive for the first time the dynamic equations with both the skew-symmetric property of the Coriolis matrix and the boundedness property of the inertia matrix for vehicle-manipulator systems with non-Euclidean joints.
\end{abstract}

Keywords: vehicle-manipulator dynamics, robot modelling, dynamic properties, singularities.

\section{INTRODUCTION}

This paper is motivated by a general concern that some frequently used properties of the inertia and Coriolis matrices for vehicle-manipulator systems are assumed true based on the proofs for other systems. We show that the proofs of these properties for fixed-base robot manipulators or single rigid bodies (vehicles) cannot be generalised to vehicle-manipulator systems directly. In fact, we show that the most commonly used dynamic equations for vehiclemanipulator systems do not possess both the boundedness and skew-symmetric properties. There is thus a need to clarify to what extent these properties are true and to find a rigorous mathematical representation of these systems for use in simulations and controller design. To this end we present a reformulation of the dynamic equations for vehicle-manipulator systems for which both the boundedness and the skew-symmetric properties are true.

Lyapunov based controllers are based on several assumptions that simplify the controller design. Some of these properties are almost universally taken for granted. In this paper we discuss two such properties that cannot be assumed true for vehicle-manipulator systems without further consideration. The first property is concerned with the boundedness of the inertia matrix $M$, i.e. if there exist lower and upper bounds on its singular values. For robot manipulators this boundedness property is addressed in Ghorbel et al. (1998) where the class of robots for which the inertia matrix is bounded is characterised. The work of Ghorbel et al. (1998) differs from our approach in that they are mainly concerned with the design of the manipulator while we are concerned with the mathematical representation. For a given robotic manipulator there may exist one mathematical representation for which the inertia matrix is bounded and another for which it is not. We find that for the most commonly used mathematical representation of vehicle-manipulator systems this property is not true.

The second property addressed is how to find a parameterisation of the Coriolis matrix $C$ so that the matrix $\dot{M}-2 C$ is skew-symmetric. Such a parameterisation is easy to find for fixed-base robots or for a single rigid body, but not always for vehicle-manipulator systems. Particularly we find that such a parameterisation is hard to find, especially together with the boundedness property.

We will focus on two important classes of vehiclemanipulator systems - underwater-manipulator systems and spacecraft-manipulator systems - but the results are general and also applicable to other vehicles. Underwatermanipulator systems are extensively treated in Antonelli (2006); Schjolberg (1996); Schjolberg and Fossen (1994) and Fossen and Fjellstad. For the choice of state variables used in most of the literature, the boundedness property does not hold for the whole configuration space, i.e. there exist isolated points where the inertia matrix becomes singular. This can, however, be dealt with by introducing a quaternion representation (Fossen, 2002). The quaternion representation is well suited for single rigid bodies, but for multibody systems the Euler angles are normally adopted. The problems regarding the Euler angle singularities are pointed out in most papers when it comes to modelling, but is often left out when dealing with Lyapunov stability. 
As a result of this the control law is not valid at isolated points in the configuration space.

Similarly, the skew-symmetric property of the Coriolis matrix is in general not treated correctly and is in most cases assumed true without any further proof. In the authors' view, this is a strong weakness because this property depends on how we choose to represent the Coriolis matrix. It is thus not sufficient to refer to an arbitrary proof of skew-symmetry, one must refer to a proof for the specific parameterisation of the Coriolis matrix chosen. Most papers on the topic refer to Antonelli (2006), Fossen and Fjellstad, de Wit et al. (1998) or Schjolberg and Fossen (1994) for this proof. However, none of these references actually show the proof. Given the velocity state $v$, Schjolberg and Fossen (1994) state that $v^{\top}(\dot{M}-2 C) v=0$, which is true, but a weaker result than skew-symmetry. This property is known as the principle of conservation of energy and is always true. This is often used to show skew-symmetry, which is not correct. Other commonly used references are taken from the fixed-base robotics literature, such as Murray et al. (1994); Sciavicco and Siciliano (2005) and Craig (1987). The proof can be found in Schjolberg (1996), but only for systems where the boundedness property does not hold. We present this proof, and correct some mistakes made, so that this proof is correctly presented for future reference.

Spacecraft are normally modelled using quaternions and the inertia matrix is thus bounded for the whole configuration manifold (Wen and Kreutz-Delgado, 1991). A Lie group formulation of the attitude of a single rigid body on $S O(3)$ that satisfies both the boundedness and the skewsymmetric property can be found using the formulation in Bullo and Lewis (2004) or Marsden and Ratiu (1999). For spacecraft-manipulator systems, however, a Langrangian approach is normally adopted and, again, the dynamics are not globally valid. Such systems are discussed in Hughes (2002); Moosavian and Papadopoulos (2007); Vafa and Dubowsky (1987) and Liang et al. (1998). As for the underwater systems, most papers concerned with modelling address this, but it is often not noted in the stability proofs (Antonelli, 2006). Also for the skew-symmetric property the most commonly used references only show this for fixed-base manipulators, such as in Murray et al. (1994); Sciavicco and Siciliano (2005) and Craig (1987). In Egeland and Pettersen (1998) the dynamics possess the skewsymmetric property and, based on the proof in Schjolberg and Fossen (1994), we show that this is also true when the dynamics are written in terms of global state variables.

\section{PROPERTIES OF THE DYNAMICS}

In this section we list some important properties of dynamical systems in matrix form that play important roles in system analysis as well as controller design. Assume a mechanical system with dynamics

$$
M(q) \ddot{q}+C(q, \dot{q}) \dot{q}=\tau
$$

where $q$ is the state of the system, $M(q)$ is the inertia matrix and $C(q, \dot{q})$ is the Coriolis and centripetal matrix. The following properties can be associated with the inertia and Coriolis matrices (Borhaug, 2008):

Property 1. (The boundedness property) The inertia matrix $M(q)$ is uniformly bounded in $q$, i.e. there exist constants $d_{1}, d_{2}$, such that

$$
0<d_{1} \leq\|M(q)\| \leq d_{2}<\infty, \quad \forall q \in \mathbb{R}^{n},
$$

where $\|\cdot\|$ is the induced norm for matrices, i.e. a maxbound on the maximum singular value and a min-bound on the minimum singular value of the matrix.

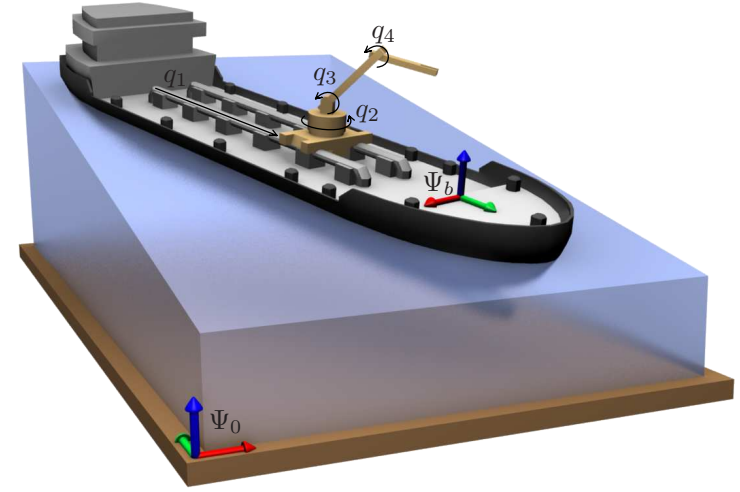

Fig. 1. Model setup for a robot mounted on a vehicle with coordinate frame $\Psi_{b}$ and inertial reference frame $\Psi_{0}$.

Property 2. (The skew-symmetric property) The matrix $(\dot{M}(q)-2 C(q, \dot{q}))$ is skew-symmetric.

Property 1 is true only when there are no singularities present in the formulation. Thus, if the Euler angles are used to represent the attitude of the vehicle, as in Fossen (2002); Schjolberg (1996); Borhaug (2008), this is not satisfied. The existence of the boundaries $d_{1}$ and $d_{2}$ is the basis of gain controller design and global Lyapunov stability and used in several manipulator control laws (Ghorbel et al., 1998). Many controllers assume the property

$$
\left\|M(q)^{-1} \hat{M}(q)-I\right\| \leq d<1, \quad \forall q \in \mathbb{R}^{n}
$$

which is automatically satisfied if $d$ is chosen as $d=\frac{d_{2}-d_{1}}{d_{2}+d_{1}}$. Property 1 guarantees that the constant $d$ is bounded and is thus important in a large class of existing control laws.

Property 2 is true for a certain parameterisation of the Coriolis matrix. Such a representation is well known for robotic manipulators on a fixed base (Murray et al., 1994; Sciavicco and Siciliano, 2005) and for vehicles with no manipulator attached (Fossen, 2002). One formulation for vehicle-manipulator systems is found in Egeland and Pettersen (1998) where the boundedness and skew-symmetric properties are both true. The formulation uses quasivelocities and the final equations resemble Kirchhoff's equations (Fossen, 2002), but for multibody systems.

The formulation presented in Egeland and Pettersen (1998) is, however, independent of the position variables of the vehicle and there is no obvious way to include these without introducing the singularities that arise in the transformation between the local and global velocity variables. The configuration of the vehicle is important in order to include terms such as gravity and buoyancy and also ocean currents which is in the form $\nu_{\text {rel }}=\nu-R_{0 b} \nu_{\text {curr }}$ where $\nu$ is the velocity in the body frame, $\nu_{\text {curr }}$ is the velocity of the ocean current in the inertial frame and $R_{0 b}$ is the configuration dependent rotation matrix. Hence, this transformation also requires the position state of the vehicle. The formulation in Egeland and Pettersen (1998) does not need the position variables of the vehicle. For more general systems the position variables may be required in the inertia matrix, and in this setting this formulation does not hold in terms of the boundedness property. This includes systems with more than one transformation that cannot be represented with generalised coordinates.

Based on these observations, we organise the paper as follows: In Section 3 we derive the dynamics of Lagrangian systems in terms of generalised coordinates which include standard fixed-base robotic manipulators and we show that Properties 1 and 2 hold. In Section 4 we derive the dynamics for single rigid bodies when the state space 
cannot be written as generalised coordinates and we show that Property 2 holds, but Property 1 does not. Section 5.1 describes the dynamics of vehicle-manipulator systems as they normally appear in literature and we show that the boundedness property does not hold. In Section 5.2 the dynamics of vehicle-manipulator systems are derived based on Egeland and Pettersen (1998) and the boundedness and skew-symmetric properties are shown. We also correct some mistakes that occur in Egeland and Pettersen (1998). In Section 5.3 we present the correct equations based on their approach and extend these to the more general case when also the position variables of the vehicle are included. We also present the explicit expressions of the matrices that do not appear in Egeland and Pettersen (1998) as well as a proof of the skew-symmetric property. In Section 5.3 we present for the first time a set of equations for where both the boundedness and skew-symmetric properties hold for generic vehicle-manipulator systems that cannot be written in terms of generalised coordinates.

\section{LAGRANGIAN DYNAMICS ON $\mathbb{R}^{n}$}

A wide range of dynamical systems can be described by the Lagrange equations (Goldstein et al., 2001)

$$
\frac{\mathrm{d}}{\mathrm{d} t}\left(\frac{\partial \mathcal{L}}{\partial \dot{q}}(q, \dot{q})\right)-\frac{\partial \mathcal{L}}{\partial q}(q, \dot{q})=\tau
$$

where $q \in \mathbb{R}^{n}$ is a vector of generalised coordinates and $\tau \in \mathbb{R}^{n}$ are the generalised forces. We note that the position variables are written as $q \in \mathbb{R}^{n}$ and the velocity variables are written as $v=\dot{q} \in \mathbb{R}^{n}$. This is a convenient choice of state variables for many systems but, as we will see later, there are also many systems for which the position and velocity variables cannot be written in this form. The Lagrangian is given by

$$
\mathcal{L}(q, \dot{q}): \mathbb{R}^{n} \times \mathbb{R}^{n} \rightarrow \mathbb{R}:=\mathcal{U}(q, \dot{q})-\mathcal{V}(q)
$$

Here, $\mathcal{U}(q, \dot{q})$ is the kinetic and $\mathcal{V}(q)$ the potential energy functions. We assume that the kinetic energy function is positive definite and in the form $\mathcal{U}(q, \dot{q}):=\frac{1}{2} \dot{q}^{\top} M(q) \dot{q}$. We can recast (4) into the equivalent form

$$
M(q) \ddot{q}+C(q, \dot{q}) \dot{q}+n(q)=\tau
$$

where $n(q):=\frac{\partial V}{\partial q}(q)$ is the vector of potential forces.

\subsection{The Boundedness Property}

Ghorbel et al. (1998) identify all manipulators for which the boundedness condition of the inertia matrix is satisfied. For a large class of manipulators, including manipulators with only revolute or only prismatic joints, this property is always satisfied. They do not address the mathematical representation of the inertia matrix. However, as long as we can use generalised coordinates to represent the state of the robot we can do this without the presence of singularities. In this case, all manipulators characterised by Ghorbel et al. (1998) to satisfy Property 1 from a design point of view, will also satisfy Property 1 from mathematical point of view, as is our main concern.

\subsection{The Skew-Symmetric Property}

For robotic manipulators represented in generalised coordinates, the Coriolis matrix is normally obtained by the Christoffel symbols of the first kind as (Murray et al., 1994)

$C(q, \dot{q}):=\left\{c_{i j}\right\}=\frac{1}{2}\left\{\sum_{k=1}^{n}\left(\frac{\partial m_{i j}}{\partial q_{k}}+\frac{\partial m_{k i}}{\partial q_{j}}-\frac{\partial m_{k j}}{\partial q_{i}}\right) \dot{q}_{k}\right\}$, where $M(q)=\left\{m_{i j}\right\}$. Given this representation it is straight forward to show that $(\dot{M}-2 C)$ is skew-symmetric and Property 2 is satisfied (Murray et al., 1994).
We note that to obtain the Coriolis matrix we multiply the position and the velocity. This only makes sense if the derivative of the position equals the velocity. This is the case for robot manipulators with revolute or prismatic joints, but not for vehicles with configuration space $S O(3)$ or $S E(3)$. The Christoffel symbols can thus not be used to derive the Coriolis matrix for such systems.

\section{VEHICLE DYNAMICS}

The dynamics of a single rigid body, such as an underwater vehicle are usually given by (Fossen, 2002)

$$
\begin{aligned}
\dot{\eta} & =J(\eta) \nu, \\
M \dot{\nu}+C(\nu) \nu+D(\nu) \nu+n(\eta) & =\tau
\end{aligned}
$$

where $\eta=\left[\begin{array}{llllll}x & y & z & \phi & \theta & \psi\end{array}\right]^{\top}$ is the position and orientation of the vehicle in the reference frame and $\nu=$ $\left[\begin{array}{llllll}u & v & w & p & q & r\end{array}\right]^{\top}$ is the linear and angular velocities in the body frame. $D(\nu)$ is the friction and damping matrix present for underwater vehicles and $n(\eta)$ represent the gravitational and buoyancy forces. The kinematics of the system (7) is given by the velocity transformation matrix

$$
J(\eta)=\left[\begin{array}{cc}
R_{b 0}(\Theta) & 0 \\
0 & T_{\Theta}(\Theta)
\end{array}\right]
$$

where $R_{b 0}(\Theta)$ is the rotation matrix and depends only on the orientation of the vessel represented by the Euler angles $\Theta=\left[\begin{array}{lll}\phi & \theta & \psi\end{array}\right]^{\top}$. $T_{\Theta}(\Theta)$ is given by (zyx-sequence)

$$
T_{\Theta}(\Theta)=\left[\begin{array}{ccc}
1 & \sin \phi \tan \theta & \cos \phi \tan \theta \\
0 & \cos \phi & -\sin \phi \\
0 & \frac{\sin \phi}{\cos \theta} & \frac{\cos \phi}{\cos \theta}
\end{array}\right] .
$$

$T_{\Theta}(\Theta)$, and thus also $J(\eta)$, is not defined for $\theta= \pm \frac{\pi}{2}$.

We can rewrite the dynamics using general coordinates $\eta$, eliminating the body frame coordinates $\nu$ from the equations. The dynamics are then written as

where

$$
\tilde{M}(\eta) \ddot{\eta}+\tilde{C}(\eta, \dot{\eta}) \dot{\eta}+\tilde{D}(\eta, \dot{\eta}) \dot{\eta}+\tilde{n}(\eta)=\tilde{\tau}
$$

$$
\begin{aligned}
\tilde{M}(\eta) & =J^{-\mathrm{\top}}(\eta) M J^{-1}(\eta) \\
\tilde{n}(\eta) & =J^{-\mathrm{\top}}(\eta) n(\eta) \\
\tilde{\tau} & =J^{-\mathrm{\top}}(\eta) \tau
\end{aligned}
$$

$\tilde{D}(\eta, \dot{\eta}) \dot{\eta}=J^{-\mathrm{T}}(\eta) D\left(J^{-1}(\eta) \dot{\eta}\right) J^{-1}(\eta)$

$\tilde{C}(\eta, \dot{\eta}) \dot{\eta}=J^{-\mathrm{T}}(\eta)\left[C\left(J^{-1}(\eta) \eta\right)-M J^{-1}(\eta) \dot{J}(\eta)\right] J^{-1}(\eta)$

\subsection{The Boundedness Property}

First note that for the system (7-8) the inertia matrix is always bounded as it is independent of the position variables. In this sense it is advantageous to formulate the dynamics in the body frame. Consider the system (12-16) and recall that $J(\eta)$ is not defined for $\theta= \pm \frac{\pi}{2}$. This is the well known Euler angle singularity for the $z y x$-sequence. The inverse mappings $T_{\Theta}^{-1}(\Theta)$ and $J^{-1}(\eta)$ are defined for all $\theta \in \mathbb{R}$ but singular for $\theta= \pm \frac{\pi}{2}$. Thus, the boundedness of the inertia matrix does not hold. We only obtain a weaker result than the one found in Property 1:

Property 3. (The weak boundedness property) The inertia matrix $M(\eta)$ is uniformly bounded in $\eta$ for $\theta$ separated from $\pm \frac{\pi}{2}$, i.e. there exists constants $d_{1}$ and $d_{2}$ such that

$$
0<d_{1} \leq\|\tilde{M}(\eta)\| \leq d_{2}<\infty, \quad \forall \eta \in \mathbb{R}^{6} \backslash\left\{|| \theta\left|-\frac{\pi}{2}\right| \geq \delta\right\}
$$

for some small $0<\delta$. We note that the lower bound $d_{1}>0$ only exists if $|\theta|$ is separated from $\frac{\pi}{2}$ by some constant $\delta$. 
When $\theta=\frac{\pi}{2}$ we have $d_{1}=0$ which gives $d=1$ and (3) does not hold. This singularity can be avoided by using the unit quaternion representation, which does not have a singularity at the cost of introducing a fourth parameter to describe the orientation. However, in computing the Euler angles from the quaternions the singularity reappears.

\subsection{The Skew-Symmetric Property}

There are many ways to choose the Coriolis matrix so that $\dot{M}-2 C$ is skew-symmetric. First note that if $M$ is constant this is true if $C$ is skew-symmetric. If $M$ is not constant, we can show this property by writing (Fossen, 2002)

$$
C(\nu)=\left[\begin{array}{cc}
0 & \widehat{M_{11} \nu_{1}}+\widehat{M_{12} \nu_{2}} \\
\widehat{M_{11} \nu_{1}}+\widehat{M_{12} \nu_{2}} & \widehat{M_{21} \nu_{1}}+\widehat{M_{22} \nu_{2}}
\end{array}\right]
$$

where $\nu=\left[\begin{array}{ll}\nu_{1}^{\top} & \nu_{2}^{\top}\end{array}\right]^{\top}$ and $\hat{\lambda}$ is the skew-symmetric matrix representation of $\lambda \in \mathbb{R}^{3}$ such that $\hat{\lambda} x=\lambda \times x$ for all $\lambda, x \in \mathbb{R}^{3}$. The expression in (17) can also be found from Kirchhoff's equations (Sagatun and Fossen, 1992) which also satisfies Property 2. Several other representations that satisfy Property 2 are found in Fossen and Fjellstad.

\section{VEHICLE-MANIPULATOR DYNAMICS}

In this section we review some commonly used approaches for modelling multibody systems and propose a new approach that has certain advantages when it comes to the boundedness and skew-symmetric properties addressed in this paper. Consider the setup of Figure 1 describing a general $n$-link robot manipulator arm attached to a freemoving base. Choose an inertial coordinate frame $\Psi_{0}$, a frame $\Psi_{b}$ rigidly attached to the moving base, and $n$ frames $\Psi_{i}$ (not shown) attached to each link $i$ at the center of mass with axes aligned with the principal directions of inertia. Finally, choose a vector $q \in \mathbb{R}^{n}$ that describes the configuration of the $n$ joints.

\subsection{The Model of Schjolberg (1996)}

In this section we present the dynamic equations as they are normally presented in the underwater robotics literature. The details can be found in Schjolberg (1996). The dynamics can be written as

$$
\begin{aligned}
\dot{\xi} & =J(\xi) \zeta, \\
M(q) \dot{\zeta}+C(q, \zeta) \zeta+D(q, \zeta) \zeta+n(\xi) & =\tau
\end{aligned}
$$

where $\xi=\left[\begin{array}{ll}\eta^{\top} & q^{\top}\end{array}\right]^{\top}, \zeta=\left[\begin{array}{ll}\nu^{\top} & \dot{q}^{\top}\end{array}\right]^{\top}, M(q) \in \mathbb{R}^{(6+n) \times(6+n)}$ is the inertia matrix, $C(q, \zeta) \in \mathbb{R}^{(6+n) \times(6+n)}$ is the Coriolis matrix and $D(\xi, \zeta) \in \mathbb{R}^{(6+n) \times(6+n)}$ is the damping matrix. The velocity transformation matrix is given by

$$
J(\xi)=\left[\begin{array}{ccc}
R_{0 b}(\Theta) & 0 & 0 \\
0 & T_{\Theta}(\Theta) & 0 \\
0 & 0 & I
\end{array}\right] \in \mathbb{R}^{(6+n) \times(6+n)} .
$$

where $I$ (no subscript) denotes the identity matrix. Similarly to the dynamics of the vehicle with no robotic arm the vehicle-manipulator equations can be written in the form of Equations (12-16) but with the velocity transformation matrix as in (20). Let $\nu_{0 i}^{0}$ denote the linear and angular velocity of body $i$ represented in the inertial frame, and $P_{i}(q) \in \mathbb{R}^{6 \times(6+n)}$ be the transformation matrix of link $i$, that gives the relation $\nu_{0 i}^{0}=P_{i}(q) \zeta$. The inertia matrix of the vehicle-manipulator system can then be written as (Egeland and Pettersen, 1998)

$$
M(q)=\sum_{i=b}^{n} P_{i}^{\top}(q) I_{i} P_{i}(q)
$$

where $I_{i} \in \mathbb{R}^{6 \times 6}$ denote the constant positive-definite diagonal inertia tensor of link $i$ expressed in $\Psi_{i}$. We note that the inertia matrix is independent of the position $\eta$ of the vehicle. The Coriolis matrix is given by

$$
C(q, \zeta)=\sum_{i=b}^{n} \dot{P}_{i}^{\top}(q) I_{i} P_{i}(q)-P_{i}^{\top}(q) W_{i}(\zeta) P_{i}(q)
$$

where $W_{i}(\zeta)$ is skew-symmetric (Schjolberg, 1996).

Alternatively we can write the dynamics in terms of the vector $\varsigma=\left[\eta^{\top}\left(x_{e}^{0}\right)^{\top}\right]^{\top} \in \mathbb{R}^{12 \times 12}$ where $x_{e}^{0}$ is the manipulator position/orientation vector in the inertial frame. We present the equations as first presented in Schjolberg (1996), but note that the Coriolis matrix should be written as (22). The dynamics can be written as

$$
\bar{M}(\eta, q) \ddot{\varsigma}+\bar{C}(\eta, q, \zeta) \dot{\zeta}+\bar{D}(\eta, q, \zeta) \dot{\zeta}+\bar{n}(\eta, q)=\bar{\tau}
$$

with the matrices as in (12-16), but with

$$
\bar{J}(\eta)=\left[\begin{array}{cc}
J(\eta) & 0 \\
\bar{R}_{b 0}(\Theta) \bar{J}_{1}(\eta) & \bar{R}_{b 0}(\Theta) \bar{J}_{2}(q)
\end{array}\right] \in \mathbb{R}^{12 \times(6+n)}
$$

where $J(\eta)$ is given by (9) and $\bar{J}_{1}$ and $\bar{J}_{2}$ satisfy

$$
\dot{x}_{e}^{b}=\bar{J}_{1}(\eta) \nu+\bar{J}_{2}(q) \dot{q}
$$

where $x_{e}^{b}$ is the end-effector position/orientation in the base frame and $\bar{R}_{b 0}(\Theta)=\operatorname{diag}\left\{R_{b 0}, R_{b 0}\right\}$.

\subsection{Multibody Dynamics in Terms of Quasi-Velocities}

In this section we derive the dynamics of a robotic manipulator mounted on a free-floating base in terms of quasivelocities. The approach is based on Egeland and Pettersen (1998). First, write the linear and angular velocities $\nu_{0 i}^{0}$ of each link $i$ represented in the inertial frame (frame 0) as

$$
\nu_{0 i}^{0}=\left[\begin{array}{c}
\nu_{0 i, v}^{0} \\
\nu_{0 i, \omega}^{0}
\end{array}\right]=\frac{\partial \nu_{0 i}^{0}}{\partial \zeta} \zeta .
$$

The dynamics are written as (Egeland and Pettersen, 1998)

$$
\sum_{i=b}^{6}\left\{\frac{\partial \nu_{0 i}^{0}{ }^{\top}}{\partial \zeta}\left[\frac{\mathrm{d}}{\mathrm{d} t}{\frac{\partial \mathcal{U}_{i}{ }^{\top}}{\partial \nu_{0 i}^{0}}}^{\top}+\left[\begin{array}{cc}
\hat{\nu}_{0 i, \omega}^{0} & 0 \\
\hat{\nu}_{0 i, v}^{0} & \hat{\nu}_{0 i, \omega}^{0}
\end{array}\right] \frac{\partial \mathcal{U}_{i}{ }^{\top}}{\partial \nu_{0 i}^{0}}\right]\right\}=\tau
$$

where $\tau=\sum_{i=b}^{6}{\frac{\partial \nu_{0 i}^{0}}{\partial \zeta}}^{\top} \tau_{i}$. We now show the explicit expressions for the matrices, which were not shown in Egeland and Pettersen (1998), and we correct an error is the expression of the Coriolis matrix. First write

$$
\frac{\mathrm{d}}{\mathrm{d} t} \frac{\partial \mathcal{U}_{i}}{\partial \nu_{0 i}^{0}}=\frac{\mathrm{d}}{\mathrm{d} t}\left(I_{i} \nu_{0 i}^{0}\right)=I_{i} \dot{\nu}_{0 i}^{0}=I_{i}\left(\frac{\partial \nu_{0 i}^{0}}{\partial \zeta} \dot{\zeta}+\frac{\partial \dot{\nu}_{0 i}^{0}}{\partial \zeta} \zeta\right)
$$

and

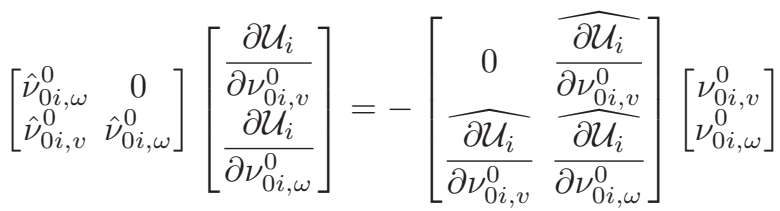

The inertia matrix is given by $(21)$ with $P_{i}(q)=\frac{\partial \nu_{0 i}^{0}}{\partial \zeta}$ and the Coriolis matrix is given by $(22)$ where $W_{i}\left(\nu_{0 i}^{0}\right)$ is the matrix on the right hand side in (29).

\subsection{General Multibody Dynamics}

In this section we extend the formulation in the previous section to include more general structures and also structures where the position of the vehicle needs to be included 
in the dynamics. The approach is based on Duindam and Stramigioli (2008) and From et al. (2009) where the dynamics of vehicle-manipulator systems are derived and the boundedness property holds. However, for the Coriolis matrix presented in From et al. (2009) the symmetry property does not hold. In this section we thus present a new formulation of the dynamic equations for vehiclemanipulator systems where both the boundedness and the symmetry properties hold and which allows us to include the vehicle configuration in the representation.

We can describe the pose of each frame $\Psi_{i}$ relative to $\Psi_{0}$ as a homogeneous transformation matrix $g_{0 i} \in S E(3)$ by

$$
g_{0 i}=\left[\begin{array}{cc}
R_{0 i} & p_{0 i} \\
0 & 1
\end{array}\right] \in \mathbb{R}^{4 \times 4}
$$

with rotation matrix $R_{0 i} \in S O(3)$ and translation vector $p_{0 i} \in \mathbb{R}^{3}$. This pose can also be described using the vector of joint coordinates $q$ as

$$
g_{0 i}=g_{0 b} g_{b i}=g_{0 b} g_{b i}(q)
$$

The base pose $g_{0 b}$ and the joint positions $q$ thus fully determine the configuration state of the robot. Similarly the spatial velocity of each link can be expressed using twists:

$$
\nu_{0 i}^{0}=\left[\begin{array}{c}
\nu_{0 i, v}^{0} \\
\nu_{0 i, \omega}^{0}
\end{array}\right]=\nu_{0 b}^{0}+\nu_{b i}^{0}=\operatorname{Ad}_{g_{0 b}}\left(\nu_{0 b}^{b}+J_{i}(q) \dot{q}\right)
$$

where $\nu_{0 i, v}^{0}$ and $\nu_{0 i, \omega}^{0}$ are the linear and angular velocities, respectively, of link $i$ relative to the inertial frame, $J_{i}(q) \in$ $\mathbb{R}^{6 \times n}$ is the geometric Jacobian of link $i$ relative to $\Psi_{b}$ and the adjoint is defined as $\operatorname{Ad}_{g}:=\left[\begin{array}{cc}R & \hat{p} R \\ 0 & R\end{array}\right] \in \mathbb{R}^{6 \times 6}$. The velocity state is thus fully determined given the twist $\nu_{0 b}^{b}$ of the base and the joint velocities $\dot{q}$. This illustrates how the kinematics of the system can be naturally described in terms of the (global) state variables $Q=\left\{g_{0 b}, q\right\}$ and $v=$ $\left\{\nu_{0 b}^{b}, \dot{q}\right\}$. We will use these observations to reformulate the relation given in (26) and rewrite the expression for kinetic energy in terms of the globally defined state variables.

Given a mechanism with coordinates formulated in this generalised form, we can write its kinetic energy as $\mathcal{U}_{k}(Q, v)=\frac{1}{2} v^{\top} M(Q) v$ with $M(Q)$ the inertia matrix in coordinates $Q$. The dynamics of this system then satisfy

$$
M(Q) \dot{v}+C(Q, v) v=\tau \text {. }
$$

From expression (32) for the twist we can derive an expression for the total kinetic energy. The kinetic energy $\mathcal{U}_{i}=\frac{1}{2}\left(\nu_{0 i}^{0}\right)^{\top} I_{i} \nu_{0 i}^{0}$ of link $i$ then follows as

$$
\begin{aligned}
\mathcal{U}_{i} & =\frac{1}{2}\left(\nu_{0 b}^{b}+J_{i}(q) \dot{q}\right)^{\top} \operatorname{Ad}_{g_{i b}}^{\top} I_{i} \operatorname{Ad}_{g_{i b}}\left(\nu_{0 b}^{b}+J_{i}(q) \dot{q}\right) \\
& =\frac{1}{2}\left[\left(\nu_{0 b}^{b}\right)^{\top} \dot{q}^{\top}\right] M_{i}(q)\left[\begin{array}{c}
\nu_{0 b}^{b} \\
\dot{q}
\end{array}\right]=\frac{1}{2} v^{\top} M_{i}(q) v
\end{aligned}
$$

with

$$
M_{i}(q):=\left[\begin{array}{cc}
\operatorname{Ad}_{g_{i b}}^{\top} I_{i} \operatorname{Ad}_{g_{i b}} & \operatorname{Ad}_{g_{i b}}^{\top} I_{i} \operatorname{Ad}_{g_{i b}} J_{i} \\
J_{i}^{\top} \operatorname{Ad}_{g_{i b}}^{\top} I_{i} \operatorname{Ad}_{g_{i b}} & J_{i}^{\top} \operatorname{Ad}_{g_{i b}}^{\top} I_{i} \operatorname{Ad}_{g_{i b}} J_{i}
\end{array}\right]
$$

where $J_{i}(q)$ is the geometric Jacobian of link $i$. The total kinetic energy of the mechanism is given by the sum of the kinetic energies of the links and the non-inertial base, i.e.,

$$
\mathcal{U}(q, v)=\frac{1}{2} v^{\top} \underbrace{\left(\left[\begin{array}{cc}
I_{b} & 0 \\
0 & 0
\end{array}\right]+\sum_{i=1}^{n} M_{i}(q)\right)}_{\text {inertia matrix } M(q)} v
$$

with $M(q)$ the inertia matrix of the total system. Note that neither $\mathcal{U}(q, v)$ nor $M(q)$ depend on the pose $g_{0 b}$ and hence the choice of inertial reference frame $\Psi_{0}$.
We see that from (35) we can reformulate the expression in (21) (Egeland and Pettersen, 1998) for the inertia matrix by writing

$$
P_{i}(q)=\left[\begin{array}{ll}
\operatorname{Ad}_{g_{i b}} & \operatorname{Ad}_{g_{i b}} J_{i}
\end{array}\right] \in \mathbb{R}^{6 \times(6+n)}
$$

and the Jacobian $J_{i}$ of link $i$ is given by

$J_{i}(q)=\left[\begin{array}{lllllll}X_{1} & \operatorname{Ad}_{g_{b 1}} X_{2} & \operatorname{Ad}_{g_{b 2}} X_{3} & \cdots & \operatorname{Ad}_{g_{b(i-1)}} X_{i} & 0_{(n-i) \times 6}\end{array}\right]$.

Similarly the Coriolis matrix can be found by (22) where $W_{i}$ is given by $(29)$ and is also well-defined.

This approach allows us to include forces such as gravity and buoyancy forces. Let $F_{g}^{i}(Q)$ be the wrench associated with the gravitational force of link $i$ with respect to coordinate frame $\Psi_{i}$. The equivalent joint torque associated with link $i$ is given by $\tau_{g}^{0}=J_{i}(q) \operatorname{Ad}_{g_{0 i}}^{\top}(Q) F_{g}^{i}(Q)$ where $J_{i}$ is the geometric Jacobian and $\operatorname{Ad}_{g_{0 i}}=\operatorname{Ad}_{g_{0 b}} \operatorname{Ad}_{g_{b i}}$ is the transformation from the inertial frame to frame $i$. We note that both $R_{0 i}$ and $\operatorname{Ad}_{g_{0 i}}$ depend on the base configuration with respect to the inertial frame. The total effect of the gravity from all the links is then given by $n(Q)=\sum_{i=1}^{n} \tau_{g}^{0}$.

We note that to obtain the complete representation of the dynamics we need to make sure we do not leave the manifold when we perform the integration. This can be done either by projecting $g_{0 b}$ onto the allowed configuration space $S E(3)$ (McLachlan and Quispel, 2006) or by using structure-preserving integration methods (Munthe-Kaas, 1998).

\subsection{Multibody Dynamics in Terms of Quasi-Coordinates}

We can also follow the generalised Lagrangian method introduced by Duindam and Stramigioli (2008) and From et al. (2009). This method gives the dynamic equations for a general mechanism described by a set $Q=\left\{Q_{i}\right\}$ of configuration states $Q_{i}$ (not necessarily Euclidean), a vector $v$ of velocity states $v_{i} \in \mathbb{R}^{n_{i}}$, and several mappings that describe the local Euclidean structure of the configuration states and their relation to the velocity states. More precisely, the neighbourhood of every state $\bar{Q}_{i}$ is locally described by a set of Euclidean coordinates $\phi_{i} \in \mathbb{R}^{n_{i}}$ as $Q_{i}=Q_{i}\left(\bar{Q}_{i}, \phi_{i}\right)$ with $Q_{i}\left(\bar{Q}_{i}, 0\right)=\bar{Q}_{i}$, and there exist differentiable matrices $S_{i}$ such that we can write $v_{i}=S_{i}\left(Q_{i}, \phi_{i}\right) \dot{\phi}_{i}$ for every $Q_{i}$. The Coriolis matrix $C(Q, v)$ can then be found in terms of $S(Q, \phi)$ and $M(Q)$. More details and proofs can be found in Duindam and Stramigioli (2008).

\subsection{The Boundedness Property}

The dynamics as presented in Schjolberg (1996) and Section 5.1 do not satisfy Property 1 . Due to the singularity there exist isolated points in the configuration space where the inertia matrix is singular. Even though this is the most common formulation of vehicle-manipulator systems in literature this fact is normally not addressed in Lyapunov stability proofs. The formulation in Egeland and Pettersen (1998) and Section 5.2 is globally valid and the inertia matrix is bounded for the whole configuration space. For systems where the configuration of non-Euclidean joints needs to be included, there does not seem to be a simple way to include the transformation between the local and global state variables without introducing singularities to the formulation. This is, however, possible with the formulation presented in Sections 5.3 and 5.4 where the inertia matrix is bounded for the whole configuration space also for non-Eucliden transformations with configuration space $S O(3)$ or $S E(3)$. 


\subsection{The Skew-Symmetric Property}

Schjolberg (1996) show that for the formulation presented in Section 5.1 the skew-symmetric property holds in bodyfixed coordinates. Based on this proof we can show that this property also holds for the approaches presented in Sections 5.2 and 5.3. First, for the Coriolis matrix given in (22) we can write

$$
\begin{aligned}
(\dot{M}-2 C)= & \frac{\mathrm{d}}{\mathrm{d} t}\left(\sum_{i=b}^{n} P_{i}^{\top}(q) I_{i} P_{i}(q)\right) \\
& -2 P_{i}^{\top}(q) I_{i} \dot{P}_{i}(q)+2 P_{i}^{\top}(q) W_{i} P_{i}(q) \\
= & 2 \sum_{i=b}^{n} P_{i}^{\top}(q) W_{i} P_{i}(q)
\end{aligned}
$$

and $(\dot{M}-2 C)$ is skew-symmetric for skew-symmetric $W_{i}$. Thus, the formulations given in Sections 5.1, 5.2 and 5.3 all satisfy the skew-symmetric property. This is not true, however, for the parameterisation in Section 5.4.

This is also true when the dynamics are given in terms of global state variables as in (11) and (23). The time derivative of the inertia matrix can be written as $\dot{\tilde{M}}=$ $J^{-\mathrm{T}}\left(\dot{M}-2 M J^{-1} \dot{J}\right) J^{-1}$ (Fossen, 1991) and we get

$$
\begin{aligned}
(\dot{\tilde{M}}-2 \tilde{C})= & J^{-\mathrm{\top}} \dot{M} J^{-1}-2 J^{-\mathrm{\top}} M J^{-1} \dot{J} J^{-1} \\
& -2 J^{-\top} C J^{-1}+2 J^{-\top} M J^{-1} \dot{J} J^{-1} \\
= & J^{-\mathrm{\top}}(\dot{M}-2 C) J^{-1}
\end{aligned}
$$

As $(\dot{M}-2 C)$ is skew-symmetric, so is $(\dot{\tilde{M}}-2 \tilde{C})$ and $(\dot{\bar{M}}-2 \bar{C})$.

\section{CONCLUSIONS}

The boundedness property of the inertia matrix and the skew-symmetric property of the Coriolis matrix both depend on the choice of mathematical representation of the matrices. The proofs of such properties thus need to be based on the particular representation chosen. In other words, a reference to a proof for a different choice of state variables or parameterisation of the matrices is not valid. In this paper we have shown that several widely used formulations of vehicle-manipulator dynamics do not possess these properties and that some of the most commonly used references in fact do not show these properties.

For several formulations of vehicle-manipulator dynamics commonly found in literature we have have studied whether the boundedness and skew-symmetric properties hold. When we find the dynamic equations to satisfy these properties we have also included the proofs for future reference. These proofs have not previously been presented correctly for vehicle-manipulator systems in the literature. Finally we propose a slightly modified version of the dynamic equations that satisfy both properties for general multibody systems.

\section{ACKNOWLEDGEMENTS}

We would like to thank Gianluca Antonelli for useful discussions and proofreading.

\section{REFERENCES}

Antonelli, G. (2006). Underwater robots. Motion and force control of vehicle-manipulator systems. Springer-Verlag.

Borhaug, E. (2008). Nonlinear Control and Synchronization of Mechanical Systems. Ph.D. thesis, Norwegian Univ. of Science and Technology, Trondheim, Norway.

Bullo, F. and Lewis, A.D. (2004). Geometric Control of Mechanical Systems: Modeling, Analysis, and Design for Simple Mechanical Control Systems. Springer.
Craig, J.J. (1987). Adaptive Control of Mechanical Manipulators. Addison-Wesley Longman Publ., Boston, MA.

de Wit, C.C., Diaz, E., and Perrier, M. (1998). Robust nonlinear control of an underwater vehicle/manipulator system with composite dynamics. International conference on robotics and automation, 452-457.

Duindam, V. and Stramigioli, S. (2008). Singularityfree dynamic equations of open-chain mechanisms with general holonomic and nonholonomic joints. IEEE Transactions on Robotics, 24(3), 517-526.

Egeland, O. and Pettersen, K.Y. (1998). Free-floating robotic systems. Control Problems in Robotics and Automation, 230, 119-134.

Fossen, T.I. and Fjellstad, O.E. (????). Nonlinear modelling of marine vehicles in 6 degrees of freedom. Int. Journal of Mathematical Modelling Systems, 1, no. 1.

Fossen, T.I. (1991). Nonlinear modeling and control of underwater vehicles. Ph.D. thesis, Department of Engineering Cybernetics, Norwegian University of Science and Technology, Trondheim, Norway.

Fossen, T.I. (2002). Marine Control Systems, 3rd printing. Marine Cybernetics, Trondheim, Norway.

From, P.J., Duindam, V., Gravdahl, J.T., and Sastry, S. (2009). Modeling and motion planning for mechanisms on a non-inertial base. International Conference of Robotics and Automation, Kobe, Japan.

Ghorbel, F., Srinivasan, B., and Spong, M.W. (1998). On the uniform boundedness of the inertia matrix of serial robot manipulators. Journal of Robotic Systems, 15.

Goldstein, H., Poole, C.P., and Safko, J.L. (2001). Classical Mechanics. Addison Wesley.

Hughes, P.C. (2002). Spacecraft Attitude Dynamics. Dover Publications.

Liang, B., Xu, Y., and Bergerman, M. (1998). Mapping a space manipulator to a dynamically equivalent manipulator. ASME Journal of Dynamic Systems, Measurement, and Control, 120.

Marsden, J.E. and Ratiu, T.S. (1999). Introduction to $\mathrm{Me}-$ chanics and Symmetry. Texts in Applied Mathematics. Springer-Verlag vol. 17.

McLachlan, R.I. and Quispel, G.R.W. (2006). Geometric integrators for odes. Journal of Physics A: Mathematical and General, 39, no. 19.

Moosavian, S.A.A. and Papadopoulos, E. (2007). Freeflying robots in space: an overview of dynamics modeling, planning and control. Robotica, 25.

Munthe-Kaas, H. (1998). Runge-kutta methods on lie groups. BIT Numerical Mathematics, 38, no. 1.

Murray, R.M., Li, Z., and Sastry, S. (1994). A Mathematical Introduction to Robotic Manipulation. CRC Press.

Sagatun, S.I. and Fossen, T.I. (1992). Lagrangian formulation of underwater vehicles. Conference of Systems, Man and Cybernetics, Charlottesville, VA, 1029-1034.

Schjolberg, I. and Fossen, T.I. (1994). Modelling and control of underwater vehicle-manipulator systems. Proceedings of the 3rd Conference on Marine Craft Maneuvering and Control, Southampton, UK, 45-57.

Schjolberg, I. (1996). Modeling and control of underwater robotic systems. Ph.D. thesis, Norwegian University of Science and Technology, Trondheim, Norway.

Sciavicco, L. and Siciliano, B. (2005). Modelling and Control of Robot Manipulators. Springer.

Vafa, Z. and Dubowsky, S. (1987). On the dynamics of manipulators in space using the virtual manipulator approach. IEEE International Conference on Robotics and Automation, North Carolina, USA.

Wen, J.T.Y. and Kreutz-Delgado, K. (1991). The attitude control problem. IEEE Transactions on Automatic Control, 30 no. 10 . 